POS PROCEEDINGS

\title{
Rapidity evolution of gluon TMDs from low to moderate $x$
}

Ian Balitsky

Theory Group, Jefferson Lab (JLAB), 12000 Jefferson Ave, Newport News, VA 23606,USA

and

Physics Dept., Old Dominion University, 4600 Elkhorn Ave, Norfolk VA 23529

E-mail: balitskyajlab.org

I discuss how the rapidity evolution of gluon transverse momentum dependent distribution changes from nonlinear evolution at small $x \ll 1$ to linear evolution at moderate $x \sim 1$.

DIS 2017

4-8 April 2017 Birmingham, UK 
A TMD factorization $[1,2,3]$ generalizes the usual concept of parton density by allowing PDFs to depend on intrinsic transverse momenta in addition to the usual longitudinal momentum fraction variable. These transverse-momentum dependent parton distributions (also called unintegrated parton distributions) are widely used in the analysis of semi-inclusive processes like semi-inclusive deep inelastic scattering (SIDIS) or dijet production in hadron-hadron collisions (for a review, see Ref. [3]). However, the analysis of TMD evolution in these cases is mostly restricted to the evolution of quark TMDs, whereas at high collider energies the majority of produced particles will be small- $x$ gluons. In this case one has to understand the transition between non-linear dynamics at small $x$ and presumably linear evolution of gluon TMDs at intermediate $x$.

In this presentation I discuss the connection between rapidity evolution of gluon TMD at low $x_{B}$ and at moderate $x_{B} \sim 1$. (The discussion is based on papers [4, 5]). We assume $k_{\perp}^{2} \geq$ few GeV ${ }^{2}$ so that we can use perturbative QCD, but otherwise $k_{\perp}$ is arbitrary and can be of order of $s$ as in the DGLAP evolution. In this kinematic region we will vary Bjorken $x_{B}$ and look how non-linear evolution at small $x$ transforms into linear evolution at moderate $x_{B}$. It should be noted that at least at moderate $x_{B}$ gluon TMDs mix with the quark ones. Here I disregard this mixing leaving the discussion of full matrix for future publications.

It is convenient to define the field-strength operator with attached light-like Wilson line:

$$
\mathscr{F}_{i}^{a \eta}\left(x_{B}, z_{\perp}\right) \equiv \frac{2}{s} \int d z_{*} e^{i x_{B} z_{*}}\left(\left[\infty, z_{*}\right]_{z}^{a m} g F_{\bullet i}^{m}\left(z_{*}, z_{\perp}\right)\right)^{\eta}
$$

where the index $\eta$ denotes the rapidity cutoff (2) for all gluon fields in this operator:

$$
A_{\mu}^{\eta}(x)=\int \frac{d^{4} k}{16 \pi^{4}} \theta\left(e^{\eta}-|\alpha|\right) e^{-i k \cdot x} A_{\mu}(k)
$$

The Sudakov variable $\alpha$ is defined as usual, $k=\alpha p_{1}+\beta p_{2}+k_{\perp}$. We define the light-like vectors $p_{1}$ and $p_{2}$ such that $p_{1}=n$ and $p_{2}=p-\frac{m^{2}}{s} n$, where $p$ is the momentum of the target particle of mass $m$. We use metric $g^{\mu v}=(1,-1,-1,-1)$ so $p \cdot q=\left(\alpha_{p} \beta_{q}+\alpha_{q} \beta_{p}\right) \frac{s}{2}-(p, q)_{\perp}$. For the coordinates we use the notations $x_{\bullet} \equiv x_{\mu} p_{1}^{\mu}$ and $x_{*} \equiv x_{\mu} p_{2}^{\mu}$ related to the light-cone coordinates by $x_{*}=\sqrt{\frac{s}{2}} x_{+}$and $x_{\bullet}=\sqrt{\frac{s}{2}} x_{-}$.

Hereafter we use the notation $\left[\infty, z_{*}\right]_{z} \equiv\left[\infty_{*} p_{1}+z_{\perp}, \frac{2}{s} z_{*} p_{1}+z_{\perp}\right]$ where $[x, y]$ stands for the straight-line gauge link connecting points $x$ and $y$. Our convention is that the Latin Lorentz indices always correspond to transverse coordinates while Greek Lorentz indices are four-dimensional.

Similarly, we define

$$
\tilde{\mathscr{F}}_{i}^{a \eta}\left(x_{B}, z_{\perp}\right) \equiv \frac{2}{s} \int d z_{*} e^{-i x_{B} z_{*}} g\left(\tilde{F}_{\bullet i}^{m}\left(z_{*}, z_{\perp}\right)\left[z_{*}, \infty\right]_{z}^{m a}\right)^{\eta}
$$

in the complex-conjugate part of the amplitude.

In this notations the unintegrated gluon TMD $\mathscr{D}\left(x_{B}, z_{\perp}, \eta\right)$ can be represented as

$$
\begin{aligned}
& \left\langle p\left|\tilde{\mathscr{F}}_{i}^{a \eta}\left(x_{B}, z_{\perp}\right) \mathscr{F}^{a i \eta}\left(x_{B}, 0_{\perp}\right)\right| p+\xi p_{2}\right\rangle \equiv \sum_{X}\left\langle p\left|\tilde{\mathscr{F}}_{i}^{a \eta}\left(x_{B}, z_{\perp}\right)\right| X\right\rangle\left\langle X\left|\mathscr{F}^{a i \eta}\left(x_{B}, 0_{\perp}\right)\right| p+\xi p_{2}\right\rangle \\
& =-4 \pi^{2} \delta(\xi) x_{B} g^{2} \mathscr{D}\left(x_{B}, z_{\perp}, \eta\right)
\end{aligned}
$$

Hereafter we use a short-hand notation

$$
\left\langle p\left|\tilde{\mathscr{O}}_{1} \ldots \tilde{\mathscr{O}}_{m} \mathscr{O}_{1} \ldots \mathscr{O}_{n}\right| p^{\prime}\right\rangle \equiv \sum_{X}\left\langle p\left|\tilde{T}\left\{\tilde{\mathscr{O}}_{1} \ldots \tilde{\mathscr{O}}_{m}\right\}\right| X\right\rangle\left\langle X\left|T\left\{\mathscr{O}_{1} \ldots \mathscr{O}_{n}\right\}\right| p^{\prime}\right\rangle
$$


where tilde on the operators in the 1.h.s. of this formula stands as a reminder that they should be inverse time ordered as indicated by inverse-time ordering $\tilde{T}$ in the r.h.s. of the above equation.

As discussed e.g. in Ref. [6], such martix element can be represented by a double functional integral

$$
\left\langle\tilde{\mathscr{O}}_{1} \ldots \tilde{\mathscr{O}}_{m} \mathscr{O}_{1} \ldots \mathscr{O}_{n}\right\rangle=\int D \tilde{A} D \tilde{\psi} D \tilde{\psi} e^{-i S_{\mathrm{QCD}}(\tilde{A}, \tilde{\psi})} \int D A D \bar{\psi} D \psi e^{i S_{\mathrm{QCD}}(A, \psi)} \tilde{\mathscr{O}}_{1} \ldots \tilde{\mathscr{O}}_{m} \mathscr{O}_{1} \ldots \mathscr{O}_{n}
$$

with the boundary condition $\tilde{A}(\vec{x}, t=\infty)=A(\vec{x}, t=\infty)$ (and similarly for quark fields) reflecting the sum over all intermediate states $X$.

We will study the rapidity evolution of the operator $\tilde{\mathscr{F}}_{i}^{a \eta}\left(x_{B}, x_{\perp}\right) \mathscr{F}_{j}^{a \eta}\left(x_{B}, y_{\perp}\right)$. In the spirit of rapidity factorization, in order to find the evolution of this operator with respect to rapidity cutoff $\eta$ (see Eq. (2)) one should integrate in the matrix element (4) over gluons and quarks with rapidities $\eta>Y>\eta^{\prime}$ and temporarily "freeze" fields with $Y<\eta^{\prime}$ to be integrated over later. (For a review, see Refs. [7, 8].) In this case, we obtain functional integral of Eq. (6) type over fields with $\eta>Y>\eta^{\prime}$ in the "external" fields with $Y<\eta^{\prime}$. In terms of Sudakov variables we integrate over gluons with $\alpha$ between $\sigma=e^{\eta}$ and $\sigma^{\prime}=e^{\eta^{\prime}}$ and, in the leading order, only the diagrams with gluon emissions are relevant - the quark diagrams will enter as loops at the next-to-leading (NLO) level.

To make connections with parton model we will have in mind the frame where target's velocity is large and call the small $\alpha$ fields by the name "fast fields" and large $\alpha$ fields by "slow" fields. As discussed in Ref. [9], the interaction of "slow" gluons of large $\alpha$ with "fast" fields of small $\alpha$ is described by eikonal gauge factors and the integration over slow fields results in Feynman diagrams in the background of fast fields which form a thin shock wave due to Lorentz contraction. However, in Ref. [9] (as well as in all small- $x$ literature) it was assumed that the characteristic transverse momenta of fast and slow fields are of the same order of magnitude. For our present purposes we need to relax this condition and consider cases where the transverse momenta of fast and slow fields do differ. In this case, we need to rethink the shock-wave approach.

Let us figure out how the relative longitudinal size of fast and slow fields depends on their transverse momenta. The typical longitudinal size of fast fields is $\sigma_{*} \sim \frac{\sigma^{\prime} s}{l_{\perp}^{2}}$ where $l_{\perp}$ is the characteristic scale of transverse momenta of fast fields. The typical distances traveled by slow gluons are $\sim \frac{\sigma s}{k_{\perp}^{2}}$ where $k_{\perp}$ is the characteristic scale of transverse momenta of slow fields. Effectively, the large- $\alpha$ gluons propagate in the external field of the small- $\alpha$ shock wave, except the case $l_{\perp}^{2} \ll k_{\perp}^{2}$ which should be treated separately since the "shock wave" is not necessarily thin in this case. Fortunately, when $l_{\perp}^{2} \ll k_{\perp}^{2}$ one can use the light-cone expansion of slow fields and leave at the leading order only the light-ray operators of the leading twist. We use the combination of shock-wave and light-cone expansions and write the interpolating formulas which describe the leading-order contributions in both cases.

Here we present only the final equation for rapidity evolution of gluon TMDs (the details can be found in Refs. $[4,5])$

$$
\begin{aligned}
& \frac{d}{d \eta}\left\langle p\left|\tilde{\mathscr{F}}_{i}^{a}\left(x_{B}, x_{\perp}\right) \mathscr{F}_{j}^{a}\left(x_{B}, y_{\perp}\right)\right| p\right\rangle^{\eta=\ln \sigma} \\
& =-\alpha_{s}\langle p| \int d^{2} k_{\perp} \operatorname{Tr}\left\{\tilde{L}_{i}^{\mu}\left(k, x_{\perp}, x_{B}\right)^{\text {light-like }} L_{\mu j}\left(k, y_{\perp}, x_{B}\right)^{\text {light-like }}\right\}
\end{aligned}
$$




$$
\begin{aligned}
&+2 \operatorname{Tr}\left\{\tilde { \mathscr { F } } _ { i } ( x _ { B } , x _ { \perp } ) \left(y_{\perp} \mid\right.\right.-\frac{p^{m}}{p_{\perp}^{2}} \mathscr{F}_{k}\left(x_{B}\right)\left(i \overleftarrow{\partial}_{l}+U_{l}\right)\left(2 \delta_{m}^{k} \delta_{j}^{l}-g_{j m} g^{k l}\right) U \frac{1}{\alpha x_{B} s+p_{\perp}^{2}} U^{\dagger} \\
&\left.+\mathscr{F}_{j}\left(x_{B}\right) \frac{\alpha x_{B} s}{p_{\perp}^{2}\left(\alpha x_{B} s+p_{\perp}^{2}\right)} \mid y_{\perp}\right) \\
&+\left(x_{\perp} \mid \tilde{U} \frac{1}{\alpha x_{B} s+p_{\perp}^{2}} \tilde{U}^{\dagger}\left(2 \delta_{i}^{k} \delta_{m}^{l}-g_{i m} g^{k l}\right)\left(i \partial_{k}-\tilde{U}_{k}\right) \tilde{\mathscr{F}}_{l}\left(x_{B}\right) \frac{p^{m}}{p_{\perp}^{2}}\right. \\
&\left.\left.+\tilde{\mathscr{F}}_{i}\left(x_{B}\right) \frac{\alpha x_{B} s}{p_{\perp}^{2}\left(\alpha x_{B} s+p_{\perp}^{2}\right)} \mid x_{\perp}\right) \mathscr{F}_{j}\left(x_{B}, y_{\perp}\right)\right\}|p\rangle+O\left(\alpha_{s}^{2}\right)
\end{aligned}
$$

where $\operatorname{Tr}$ is a trace in the adjoint representation. Here $\mathscr{F}_{j}^{a}\left(x_{B}, y_{\perp}\right)$ is so-called Lipatov vertex - the amplitude of the emission of a real gluon by the operator $\mathscr{F}_{i}^{a}$

$$
\begin{aligned}
L_{\mu i}^{a b}\left(k, y_{\perp}, x_{B}\right)^{\text {light-like }} & \\
= & g\left(k_{\perp} \mid \mathscr{F}^{j}\left(x_{B}+\frac{k_{\perp}^{2}}{\alpha s}\right)\left\{\frac{\alpha x_{B} s g_{\mu i}-2 k_{\mu}^{\perp} k_{i}}{\alpha x_{B} s+k_{\perp}^{2}}\left(k_{j} U+U p_{j}\right) \frac{1}{\alpha x_{B} s+p_{\perp}^{2}} U^{\dagger}\right.\right. \\
& \left.\left.-2 k_{\mu}^{\perp} U \frac{g_{i j}}{\alpha x_{B} s+p_{\perp}^{2}} U^{\dagger}-2 g_{\mu j} U \frac{p_{i}}{\alpha x_{B} s+p_{\perp}^{2}} U^{\dagger}+\frac{2 k_{\mu}^{\perp}}{k_{\perp}^{2}} g_{i j}\right\} \mid y_{\perp}\right)^{a b}+O\left(p_{2 \mu}\right)
\end{aligned}
$$

where the operator $\mathscr{F}_{i}(\beta)$ is defined as usual

$$
\left(k_{\perp}\left|\mathscr{F}_{i}(\beta)\right| y_{\perp}\right) \equiv \frac{2}{s} \int d y_{*} e^{i \beta y_{*}-i(k, y)_{\perp}} \mathscr{F}_{i}\left(y_{*}, y_{\perp}\right)
$$

It is worth noting that at $x_{B}=0$ this vertex agrees with the one obtained in Ref. [11].

It is easy to see that our formula for the evolution kernel (7) smoothly interpolates between the $k_{T}$-factorization and TMD-factorization cases. Indeed, in the framework of the usual small- $x$ approximation $\beta_{B}$ is neglected so the corresponding "small-x" gluon TMD looks like

$$
\mathscr{F}_{i}^{a}\left(z_{\perp}, 0\right)=U_{i}^{a}\left(z_{\perp}\right) \equiv-2 i \operatorname{tr}\left\{t^{a} U \partial_{i} U^{\dagger}\right\}
$$

and Eq. (7) reduces to the non-linear equation

$$
\begin{aligned}
& \frac{d}{d \eta} \tilde{U}_{i}^{a}\left(z_{2}\right) U_{i}^{a}\left(z_{1}\right) \\
& =-\frac{g^{2}}{8 \pi^{3}} \operatorname{Tr}\left(-i \partial_{i}^{z_{2}}+\tilde{U}_{i}^{z_{2}}\right)\left[\int d^{2} z_{3}\left(\tilde{U}_{z_{2}} \tilde{U}_{z_{3}}^{\dagger}-1\right) \frac{z_{12}^{2}}{z_{13}^{2} z_{23}^{2}}\left(U_{z_{3}} U_{z_{1}}^{\dagger}-1\right)\right]\left(i \overleftarrow{\partial}_{i}^{z_{1}}+U_{i}^{z_{1}}\right)
\end{aligned}
$$

where all indices are 2-dimensional and $\operatorname{Tr}$ stands for the trace in the adjoint representation. It is easy to see that the expression in the square brackets is actually the BK kernel for the doublefunctional integral for cross sections [7, 10].

On the other hand, if $\beta_{B} \sim 1$ so that $\alpha \beta_{B} S \gg p_{\perp}^{2}$ we get a linear equation

$$
\begin{aligned}
& \left\langle\tilde{\mathscr{F}}_{j}^{a}\left(z_{\perp}, \beta_{B}\right) \mathscr{F}^{a j}\left(z_{\perp}^{\prime}, \beta_{B}\right)\right\rangle \\
& =-\frac{g^{2} N_{c}}{\pi} \int_{\sigma_{2}}^{\sigma_{1}} \frac{d \alpha}{\alpha} \int \frac{d^{2} p}{p^{2}}\left[1-e^{i\left(p, z-z^{\prime}\right)_{\perp}}\right]\left\langle\tilde{\mathscr{F}}^{a i}\left(z_{\perp}^{\prime}, \beta_{B}\right) \mathscr{F}_{i}^{a}\left(z_{\perp}, \beta_{B}\right)\right\rangle
\end{aligned}
$$

which can be rewritten as a linear equation

$$
\frac{d}{d \eta} \mathscr{D}\left(x_{B}, z_{\perp}, \eta\right)=-\frac{\alpha_{s} N_{c}}{\pi^{2}} \mathscr{D}\left(x_{B}, z_{\perp}, \eta\right) \int \frac{d^{2} p}{p^{2}}\left[1-e^{i(p, z)_{\perp}}\right]
$$


We see that the IR divergence at $p_{\perp}^{2} \rightarrow 0$ cancels while the UV divergence in the virtual correction should be cut from above by the condition $p_{\perp}^{2}<\sigma s$ following from Eq. (7). Actually, at $x_{B} \sim 1$ there will be logarithmical region $e^{\eta} m \sqrt{s} \gg p_{\perp}^{2} \gg m^{2}$ so one has to sum up leading logarithms $\left(\alpha_{s} \eta\right)^{n}$ in the evolution kernel Eq. (12) after which the kernel should reproduce the usual Sudakov double logarithms. From Eq. (7) it is clear that the transition between linear evolution (12) and the non-linear evolution (11) occurs at $x_{B}=\beta_{B} \sim \frac{m^{2}}{s}$.

In addition, at $x_{\perp}=y_{\perp}$ (light-cone DIS limit) one obtains from Eq. (7)

$$
\begin{aligned}
& \frac{d}{d \eta}\left\langle p\left|\tilde{\mathscr{F}}_{i}^{n}\left(\beta_{B}, x_{\perp}\right) \mathscr{F}^{i n}\left(\beta_{B}, x_{\perp}\right)\right| p\right\rangle^{\eta} \\
& =\frac{\alpha_{s}}{\pi} N_{c} \int_{0}^{\infty} d \beta\left\{\theta\left(1-\beta_{B}-\beta\right)\left[\frac{1}{\beta}-\frac{2 \beta_{B}}{\left(\beta_{B}+\beta\right)^{2}}+\frac{\beta_{B}^{2}}{\left(\beta_{B}+\beta\right)^{3}}-\frac{\beta_{B}^{3}}{\left(\beta_{B}+\beta\right)^{4}}\right]\right. \\
& \left.\times\left\langle p\left|\tilde{\mathscr{F}}_{i}^{n}\left(\beta_{B}+\beta, x_{\perp}\right) \mathscr{F}^{n i}\left(\beta_{B}+\beta, x_{\perp}\right)\right| p\right\rangle^{\ln \sigma^{\prime}}-\frac{\beta_{B} \beta^{-1}}{\beta_{B}+\beta}\left\langle p\left|\tilde{\mathscr{F}}_{i}^{n}\left(\beta_{B}, x_{\perp}\right) \mathscr{F}^{i n}\left(\beta_{B}, x_{\perp}\right)\right| p\right\rangle^{\ln \sigma^{\prime}}\right\}
\end{aligned}
$$

which is equivalent to DGLAP equation in the leading log approximation. Thus, our equation (7) smoothly interpolates between DGLAP, Sudakov and low- $x$ limits.

This work was supported by contract DE-AC05-06OR23177 under which the Jefferson Science Associates, LLC operate the Thomas Jefferson National Accelerator Facility, and by the grant DE-FG02-97ER41028.

\section{References}

[1] J. C. Collins, D. E. Soper Nucl. Phys. B194, 445 (1982).

[2] X. Ji, Jian-Ping Ma, and F. Yuan, Phys. Rev. D71, 034005 (2005).

[3] J. C. Collins, Foundations of Perturbative QCD (Cambridge University Press, Cambridge, 2011).

[4] I. Balitsky and A. Tarasov, JHEP 1510, 017 (2015).

[5] I. Balitsky and A. Tarasov, JHEP 1606, 164 (2016).

[6] I. Balitsky and V.M. Braun, Phys. Lett. B 222, 121 (1989); Nucl. Phys. B361, 93 (1991); Nucl. Phys. B380, 51 (1992).

[7] I. Balitsky, "High-Energy QCD and Wilson Lines", In *Shifman, M. (ed.): At the frontier of particle physics, vol. 2*, p. 1237-1342 (World Scientific, Singapore, 2001) [hep-ph/0101042].

[8] I. Balitsky, "High-Energy Ampltudes in the Next-to-Leading Order", in "Subtleties in Quantum Field Theory", ed D. Diakonov, (PNPI Publishing Dept., 2010) arXiv:1004.0057 [hep-ph].

[9] I. Balitsky, Nucl. Phys. B463, 99 (1996); Phys. Rev. D60, 014020 (1999).

[10] I. Balitsky, "Operator expansion for diffractive high-energy scattering”, [hep-ph/9706411].

[11] I. Balitsky, Phys. Rev. D70, 114030 (2004). 